Original Paper http://ajol.info/index.php/ijbcs http://indexmedicus.afro.who.int

\title{
Caractérisation et utilisation des eaux usées en horticulture dans la ville de Ouagadougou au Burkina Faso
}

\author{
Delphine Bernadette OUEDRAOGO ${ }^{1,2 *}$, Zacharia GNANKAMBARY ${ }^{1}$, \\ Hassan Bismarck NACRO ${ }^{3}$ et Michel Papaoba SEDOGO $^{1}$
${ }^{1}$ Institut de l'Environnement et de Recherches Agricoles (INERA). Laboratoire Sol-Eau-Plante 01 BP 476 Ouagadougou 01, Ouagadougou, Burkina Faso. Durable (IGEDD) 03 BP 7021 Ouagadougou 03, Burkina Faso.
${ }^{3}$ Université Nazi Boni, Institut du Développement Rural (IDR), Laboratoire d'Etude et de Recherche sur la Fertilité du sol (LERF) 01 BP 1091 Bobo-Dioulasso 01, Burkina Faso.
*Auteur correspondant; E-mail: delberno@gmail.com \\ ${ }^{2}$ Université Ouaga I Professeur Joseph KI-ZERBO, Institut de Génie de l'Environnement et du Développement
}

\section{RESUME}

Dans la ville de Ouagadougou, Capitale du Burkina Faso, la pratique de l'horticulture connaît un essor crucial. Elle est menée autour de tout point d'eau accessible, à l'intérieure comme à la périphérie de la ville. L'objectif de notre étude est de caractériser les eaux utilisées et d'évaluer les effets de l'utilisation de ces eaux sur la qualité des sols exploités. La méthodologie utilisée a consisté à réaliser une enquête auprès des exploitants et des analyses physico- chimiques des échantillons d'eau et de sols. Les investigations ont montré que $75 \%$ des exploitants ont recours aux eaux usées pour leurs activités. Cependant les acteurs sont en majorité analphabètes et n'appréhendent pas les risques liés à l'utilisation de ces eaux. Les résultats analytiques montrent une pollution des eaux de la Station d'Epuration des eaux usées et du Canal de la Maison d'Arrêt et de Correction de Ouagadougou ; ce qui indique un traitement insuffisant des eaux. Elles sont chargées de métaux lourds $\mathrm{Cu}$ et $\mathrm{Zn}$ qui peuvent constituer un risque de santé publique. L'utilisation des eaux usées augmente les teneurs du sol en phosphore total et en potassium et contribue à améliorer la fertilité des sols et rehausser les rendements des cultures.

(C) 2018 International Formulae Group. All rights reserved.

Mots clés: Irrigation, sols, agriculture, santé, urbain.

\section{Characterization and use of wastewater in horticulture in Ouagadougou, Burkina Faso}

\begin{abstract}
In the city of Ouagadougou, capital of Burkina Faso, the practice of horticulture is booming. It is conducted around any accessible water point, both inside and on the outskirts of the city. The objective of our study is to characterize the water used and to evaluate the effects of the use of these waters on the quality of the exploited soils. The methodology used consisted of carrying out a survey of the operators and physicochemical analyzes of the water and soil samples. The investigations showed that $75 \%$ of the farmers use wastewater for their activities. However, the majority are illiterate actors and do not apprehend the risks associated with the
\end{abstract}


use of these waters. The analytical results show water pollution of the Wastewater Treatment Plant and the Canal of Ouagadougou Detention and Correction House; which indicates insufficient treatment of the water. They are loaded with heavy metals $\mathrm{Cu}$ and $\mathrm{Zn}$ which can constitute a risk of public health. The use of wastewater increases soil levels of total phosphorus and potassium and helps to improve soil fertility and enhance crop yields.

(C) 2018 International Formulae Group. All rights reserved.

Keywords: Irrigation, soil, agriculture, health, urban.

\section{INTRODUCTION}

De nos jours, près de $10 \%$ de la population mondiale consomment des aliments produits en utilisant des eaux non traitées pour l'irrigation en zone urbaine et périurbaine (Scott et al., 2011; Christine, 2013; Tidjani et al., 2018). Cette proportion est plus importante en Afrique Sub Saharienne où 50 et $90 \%$ des légumes consommés par les citadins sont produits dans les zones urbaines ou périurbaines principalement avec des eaux usées (Drechsel et al., 2006; Atidegla et al., 2017). De plus, la croissance démographique des villes africaines engendre une augmentation des besoins en alimentation notamment des produits horticoles. Cependant, dans un contexte de pauvreté des sols, de manque d'eau d'irrigation en milieu urbain et périurbain, le recours aux eaux usées souvent non traitées ou mal traitées est appelé à augmenter, tant en volume qu'en superficie irriguée.

Les eaux usées sont importantes pour de nombreux agriculteurs particulièrement sous les climats arides et semi-arides (OMS, 2012; Atinkpahoun et al., 2018). Ces eaux permettent la production de légumes, de céréales et de fruits en toutes saisons et à moindre coût car, cette eau n'est pas payante. En Afrique de l'Ouest, à l'instar des villes comme Bamako au Mali et Dakar au Sénégal (Bah, 2014), 26,7\% de la population active urbaine de Ouagadougou au Burkina Faso, s'adonne à l'agriculture (MINEFID, 2016). La ville de Ouagadougou est en grande partie approvisionnée en produits maraichers et en arbres cultivés en utilisant les eaux des barrages, des puits et des forages mais surtout les eaux usées dont la qualité reste douteuse.
Diarra (2008) et Sou (2009) ont relevé la mauvaise qualité de ces eaux, des insuffisances dans le respect des normes régissant l'utilisation de ces eaux afin de préserver l'environnement et la santé humaine au Burkina Faso. Toutefois, des arguments scientifiques montrant la mauvaise qualité de ces eaux au niveau de la production horticole dans la ville de Ouagadougou n'ont pas été suffisamment rapportés (Ouédraogo et al., 2001; Kédowidé, 2010).

Notre étude a pour objectif de caractériser les eaux usées utilisées dans la culture des légumes, des fruits et des plantes d'ornement dans la ville de Ouagadougou et d'évaluer leurs effets sur la qualité des sols exploités.

\section{MATERIEL ET METHODES Milieu d'étude}

L'étude a été conduite en 2012 dans la ville Ouagadougou $\left(12^{\circ} 21^{\prime} 58^{\prime \prime} \mathrm{N}\right.$, $1^{\circ} 31^{\prime} 05^{\prime}$ 'O), capitale du Burkina Faso (Figure 1). La ville est située dans la zone soudano-sahélienne (Fontes et Guinko, 1995) et a une saison des pluies unimodale de 5 mois environ (juin à octobre) avec une pluviométrie moyenne annuelle comprise entre 600 et 900 mm (SP/CONAGESE-REEB2, 2002). La ville couvre une superficie de $2805 \mathrm{~km}^{2}$ avec une population en pleine expansion estimée en 2001 à 1238015 et 2429718 en 2014 (INSD, 2015).

La ville est drainée par des petits affluents du Massili, un des bras du Nakanbé. L'affluent principal qui la traverse est coupé par le barrage de Boulmiougou au Sud-Ouest de la ville et les barrages $n^{\circ} 1, n^{\circ} 2, n^{\circ} 3$ qui se succèdent au Nord et constituent le réceptacle 
d'une partie de ces eaux pluviales (Ouandaogo, 2008). Les autres sources d'eau sont constituées des eaux souterraines accessibles à partir des forages et des puits.

Les sols rencontrés dans la ville de Ouagadougou, sont essentiellement de types ferrugineux tropicaux, reposant sur des granites fissurés. Ils se caractérisent aussi par leurs faibles teneurs en éléments minéraux, en particulier en potassium, en phosphore et leur structure fragile très sensible à l'érosion (Zida, 2009). La Figure 1 présente la situation de la ville de Ouagadougou et la localisation des sites de l'étude.

\section{Méthodes \\ Enquête de terrain}

L'enquête de terrain a consisté en des investigations menées dans six (6) sites répartis dans les Arrondissements 2, 3, 4, 11 et 12 de Ouagadougou. Ces sites d'études ont été choisis suivant l'intensité de l'horticulture, la source d'eau utilisée pour l'irrigation, la pratique permanente des activités et la taille des espaces horticoles. Plus de neuf cent (900) producteurs permanents évoluent dans les activités horticoles autour des points d'eau dans les sites concernés. Le nombre des exploitants par site, présenté dans le Tableau 1 , est généralement fonction de la superficie disponible, de la qualité et de la quantité d'eau ainsi que de l'emplacement du site.

Des focus groupes ont permis de consulter toutes les personnes participant à l'horticulture dans la zone. Du fait du nombre très important des exploitants, de leur petite superficie et de leur similarité du point de vue des types de sols, des précédents culturaux et des itinéraires techniques des cultures, nous avons, suivant un choix raisonné sélectionné 75 personnes représentatives de la caractérisation des sites et des exploitations. Au total, 31 interviews ont été réalisées dont 19 individuelles et 12 sous forme de focus groupes.

Les informations collectées ont porté sur le choix des espaces de production, la répartition des producteurs selon leurs activités (production de plants, culture maraichère, autres), les spéculations, la période de production, les facteurs et matériel de production, les eaux et les terreaux utilisés, les sources des eaux et du terreau, les périodes et fréquences des eaux utilisées, etc.

\section{Echantillonnage des eaux et des sols}

Les échantillons des eaux ont été prélevés sur l'ensemble des sites, à plusieurs endroits représentatifs. Ils ont été conditionnés dans des bouteilles stérilisées d'un demi-litre, conservées dans des glacières réfrigérées et acheminées au laboratoire pour analyse.

Les échantillons de sols ont été prélevés à la profondeur de $0-20 \mathrm{~cm}$ sur l'ensemble des sites. Le Tableau 1 présente la source des eaux usées et les spéculations pratiquées selon les sites de l'étude.

\section{Analyse des eaux}

Les analyses physico-chimiques et biologiques des échantillons d'eau ont porté sur le $\mathrm{pH}$, le cuivre $(\mathrm{Cu})$, le zinc $(\mathrm{Zn})$, l'aluminium ( $\mathrm{Al}$ ), le nickel $(\mathrm{Ni})$, les matières en suspension, la Demande Biochimique en Oxygène (DBO), la Demande Chimique en Oxygène (DCO), les sels dissouts. Les échantillons d'eaux ont été analysés en tenant compte des normes de qualité des eaux en vigueur au Burkina Faso. Le $\mathrm{pH}$ des échantillons d'eau a été mesuré selon la norme NF T 90-008 à l'aide d'un pH-mètre. La détermination des différents ions a été faite à l'aide d'un spectromètre d'absorption atomique à flamme suivant les normes FD T 90-112 pour le $\mathrm{Zn}$ et le $\mathrm{Cu}$, la FD T 90-119 pour Al, et la NF EN ISO 14911/ FD T 90112.pour Ni. La DBO et la DCO ont été déterminés selon les normes NF EN 1899-1 et NF T 90-101, respectivement.

\section{Analyse des sols}

Pour les analyses physico-chimiques des échantillons de sols, les paramètres qui ont été déterminés sont le $\mathrm{pH}$ eau et le $\mathrm{pH}$ $\mathrm{KCl}$, le Carbone total, l'Azote total (N-total), le P-total, le K-total. Le pH a été mesuré par la méthode électro métrique dans une suspension formée de $20 \mathrm{~g}$ de sol dans $50 \mathrm{ml}$ d'eau pour le $\mathrm{pH}$ eau et $20 \mathrm{~g}$ de sol dans $50 \mathrm{ml}$ 
de $\mathrm{KCl} 1 \mathrm{~N}$ pour le $\mathrm{pH} \mathrm{KCl}$.

La détermination du Carbone total a été faite par la méthode de Walkley \& Black (1934). Pour la détermination de N-total, Ptotal et K-total, les échantillons de sol ont d'abord été minéralisés à chaud, avec un mélange $\mathrm{H}_{2} \mathrm{SO}_{4}-\mathrm{Se}-\mathrm{H}_{2} \mathrm{O}_{2}$. Par la suite, les teneurs en $\mathrm{N}$-total et $\mathrm{P}$-total ont été déterminées dans les minéralisâts, à l'aide d'un colorimètre automatique. Le K-total a été déterminé à l'aide d'un photomètre de flamme. L'appréciation de la qualité des sols a été faite par comparaison des valeurs des paramètres mesurés par rapport aux normes d'interprétation des paramètres chimiques du sol et aux normes d'interprétation de l'acidité du sol élaborées par le Bureau National des Sols du Burkina Faso (BUNASOLS, 1990).

\section{Analyse statistique}

Le traitement des données a été réalisé grâce au logiciel statistique XLSTAT version 2017.6 (disponible à l'adresse http://www.xlstat.com). Les données collectées ont été soumises à une analyse de variance (ANOVA), et les tests de comparaison des moyennes ont été effectués à partir du modèle linéaire général (GLM) suivant le test de Tukey au seuil de 5\%.

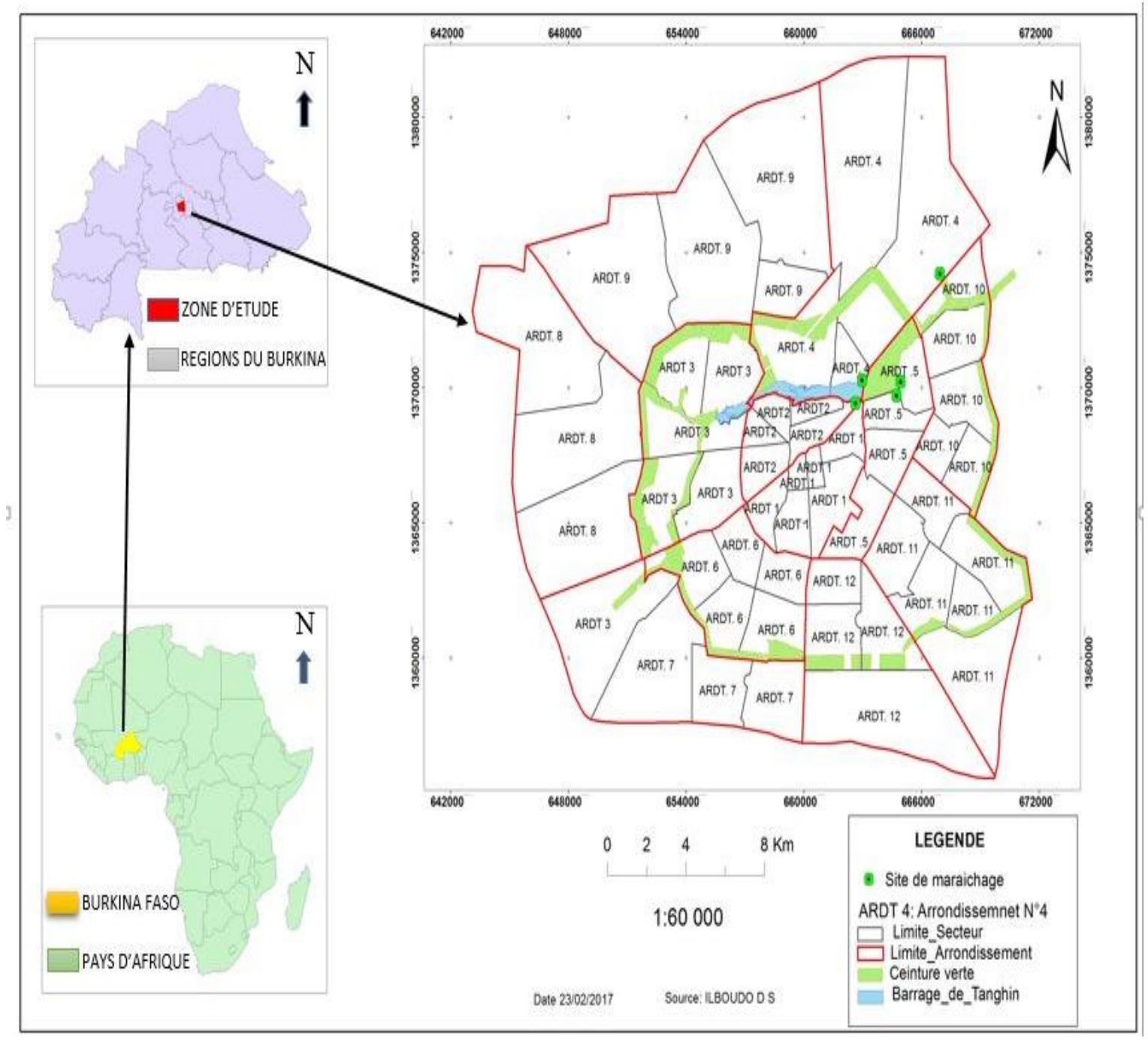

Figure 1: Situation de la ville de Ouagadougou et localisation des sites de l'étude. 
Tableau 1: Source des eaux, nombre d'exploitants et spéculations pratiquées.

\begin{tabular}{|c|c|c|c|c|c|c|}
\hline $\begin{array}{l}\text { Situation } \\
\text { géographique } \\
\text { des sites }\end{array}$ & $\begin{array}{l}\text { Identification } \\
\text { des sites }\end{array}$ & $\begin{array}{l}\text { Nombre } \\
\text { estimé } \\
\text { d'exploitants } \\
\text { présents }\end{array}$ & $\begin{array}{l}\text { Source } \\
\text { des eaux }\end{array}$ & Type d'eaux & Secteur horticole & $\begin{array}{l}\text { Spéculation } \\
\text { maraichères }\end{array}$ \\
\hline $\begin{array}{l}\text { Site } 1 \text { (secteurs } \\
\text { 19) }\end{array}$ & $\begin{array}{l}\text { STEP: - Aval } \\
\text { aménagement; - } \\
\text { Parcelles } \\
\text { aménagées de } \\
\text { la zone 3; } \\
\text { - Parcelles } \\
\text { aménagées de } \\
\text { la zone } 4\end{array}$ & 400 & STEP & $\begin{array}{l}\text { Eaux } \\
\text { canalisées à } \\
\text { partir de la } \\
\text { STEP et } \\
\text { provenant } \\
\text { des: } \\
\text { - Egouts } \\
\text { publics; } \\
\text { - Eaux usées } \\
\text { domestiques } \\
\text { - Eaux de } \\
\text { refroidissem } \\
\text { nt }\end{array}$ & Maraîchage & $\begin{array}{l}\text { Epinard, } \\
\text { Corchorussp. }( \\
\text { Interdiction de } \\
\text { produire des plantes } \\
\text { consommées crues) }\end{array}$ \\
\hline $\begin{array}{l}\text { Site } 2 \text { (secteurs } \\
\text { 22) }\end{array}$ & $\begin{array}{l}\text { Canal de la } \\
\text { MACO }\end{array}$ & 10 & $\begin{array}{l}\text { MACO et } \\
\text { quartier de } \\
\text { la zone du } \\
\text { bois }\end{array}$ & $\begin{array}{l}\text { Egouts } \\
\text { publics et } \\
\text { eaux usées } \\
\text { domestiques } \\
\text { normales. }\end{array}$ & $\begin{array}{l}\text { Maraîchage; pépinière } \\
\text { et floriculture }\end{array}$ & Amarante \\
\hline $\begin{array}{l}\text { Site } 3 \text { (Secteur } \\
21)\end{array}$ & $\begin{array}{l}\text { Canal de la } \\
\text { MACO: côté } \\
\text { Tan Aliz }\end{array}$ & 50 & $\begin{array}{l}\text { Tan Aliz; } \\
\text { MACO; } \\
\text { Usines de } \\
\text { Kossodo }\end{array}$ & $\begin{array}{l}\text { Egouts } \\
\text { publics ; } \\
\text { eaux usées } \\
\text { domestiques } \\
\text { normales; } \\
\text { eaux de } \\
\text { refroidissem } \\
\text { nt des usines; } \\
\text { eaux de } \\
\text { traitement de } \\
\text { tannerie }\end{array}$ & $\begin{array}{l}\text { Maraîchage; pépinière } \\
\text { et floriculture }\end{array}$ & Amarante \\
\hline $\begin{array}{l}\text { Site } 4 \text { (Secteur } \\
\text { 10) }\end{array}$ & $\begin{array}{l}\text { Barrage } \quad n^{\circ} 3 \\
\text { côté Sud }\end{array}$ & 300 & $\begin{array}{l}\text { Centre- } \\
\text { ville; } \\
\text { barrage } \\
n^{\circ} 3\end{array}$ & \begin{tabular}{lr}
\multicolumn{2}{l}{ Infiltration } \\
latérale & des \\
eaux & du \\
barrage & $\mathrm{n}^{\circ} 3 ;$ \\
égouts & de \\
l'hôpital & \\
Yalgado & \\
\end{tabular} & $\begin{array}{l}\text { Maraîchage; pépinière } \\
\text { et floriculture }\end{array}$ & $\begin{array}{l}\text { Aubergine, } \\
\text { carotte. }\end{array}$ \\
\hline $\begin{array}{l}\text { Site } 5 \text { (Secteur } \\
53 \text { ) }\end{array}$ & $\begin{array}{l}\text { Canal de Ouaga } \\
2000\end{array}$ & 100 & $\begin{array}{l}\text { Quartier } \\
\text { de la patte } \\
\text { d'Oie; } \\
\text { quartier } \\
\text { Ouaga } \\
2000\end{array}$ & $\begin{array}{l}\text { Eaux usées } \\
\text { domestiques } \\
\text { normales } \\
\end{array}$ & $\begin{array}{l}\text { Maraîchage; pépinière } \\
\text { et floriculture }\end{array}$ & $\begin{array}{l}\text { Aubergine, chou, } \\
\text { gombo, laitue, maïs }\end{array}$ \\
\hline $\begin{array}{l}\text { Site } 6 \text { (Secteurs } \\
\text { 17) }\end{array}$ & $\begin{array}{l}\text { Barrage } \quad n^{\circ} 3 \\
\text { côté Nord }\end{array}$ & 200 & $\begin{array}{l}\text { Quartier } \\
\text { Tanghin, } \\
\text { barrage } \\
\mathrm{n}^{\circ} 3 \text {, hôtel } \\
\text { Silmandé }\end{array}$ & $\begin{array}{l}\text { Infiltration } \\
\text { latérale des } \\
\text { eaux du } \\
\text { barrage } \mathrm{n}^{\circ} 3 \text {, } \\
\text { Puits/Forage, } \\
\text { eaux usées } \\
\text { hôtelières }\end{array}$ & $\begin{array}{l}\text { Maraîchage, pépinière, } \\
\text { floriculture }\end{array}$ & Chou, salade \\
\hline
\end{tabular}




\section{RESULTATS}

\section{Types de production}

Les enquêtes ont montré que le maraîchage et la production de plants sont pratiqués respectivement par $50 \%$ et $35 \%$ des horticulteurs. Seulement $15 \%$ de ces acteurs pratiquent des activités connexes telles que la collecte de terreaux, la confection des bouquets de fleurs, l'aménagement des espaces verts ou des parterres.

Sur l'ensemble des sites étudiés, les productions horticoles ont concerné les arbres et arbustes fruitiers, les arbres ornementaux et ombragées, les fleurs et les cultures maraîchères. Les types de spéculations sont également présentés dans le Tableau 1.

A l'exception des sites spécifiques de la STEP et de la MACO qui n'ont pratiqué uniquement que le maraîchage et la production des plants en pépinière respectivement, la plupart des sites $(67 \%)$ ont combiné le maraichage et la production des plants en pépinière. Plus de la moitié des enquêtés $(51,61 \%)$ ont indiqué que la production des plants dans les pépinières présente une plus haute valeur ajoutée et moins de travail que la culture maraîchère.

\section{Types et qualité des eaux utilisées Types d'eaux utilisées}

Les eaux utilisées sur les sites d'étude sont essentiellement usées dans $90,32 \%$ des cas (Tableau 1). Il s'agit des eaux domestiques normales, des eaux issues des égouts publics, des eaux de refroidissement des usines et des eaux issues des infiltrations latérales des barrages. Les eaux de forages et de puits divers sont utilisées dans $9,68 \%$ des cas.

\section{Propriétés physico-chimiques et biologique des eaux utilisées}

Les propriétés physico-chimiques et biologiques des eaux utilisées dans les sites d'étude sont consignées dans le Tableau 2.
Les eaux utilisées dans les plus grands espaces de production ont un $\mathrm{pH}$ élevé et compris entre 7,1 (Barrage $n^{\circ} 3$ côté Sud) et 8,3 (STEP Kossodo Polsgo (aval aménagée et parcelles zone 3), Canal MACO Tan Aliz).

L'eau du Canal de Ouaga 2000 a été la plus chargée avec $280 \mathrm{mg} / \mathrm{l}$ contre $60 \mathrm{mg} / \mathrm{l} \mathrm{de}$ matières en suspension et celle du Barrage $\mathrm{n}^{\circ} 3$ Nord-eau de Puits a été la moins chargée avec $37 \mathrm{mg} / \mathrm{l}$ de matières en suspension. Au niveau de tous les sites les valeurs sont conformes aux normes d'utilisation des eaux usées en agriculture et à celles de déversement des eaux usées dans les eaux de surfaces.

Les teneurs en matières en suspension ont été supérieures aux normes de déversement des eaux usées dans les eaux de surface et d'utilisation des eaux usées en agriculture. Les teneurs en $\mathrm{Zn}$ des eaux de tous les sites ont été très élevées surtout pour les sites du Barrage $n^{\circ} 3$ côté Sud et du Barrage $n^{\circ} 3$ côté Nord $B$ qui ont été de 30 et 40 fois supérieur à la norme d'utilisation en agriculture. Par contre, pour les eaux de la STEP, la teneur en Zn n'a été que de 2 fois cette norme. Les teneurs en $\mathrm{Cu}$ ont été plus élevées dans les eaux usées de la STEP $(0,87$ $\mathrm{mg} / \mathrm{l}$ ) et plus faible dans celles du Canal Ouaga 2000 et du Canal du Barrage n ${ }^{\circ} 3$ Nordeau de Puits $(<0,04 \mathrm{mg} / \mathrm{l})$.

Pour les eaux usées provenant du Canal du Barrage $n^{\circ} 3$ côté Sud, du Canal du Barrage $\mathrm{n}^{\circ} 3$ Nord, la DBO a été inférieure à la norme d'utilisation en agriculture $(60 \mathrm{mg}$ $\mathrm{O}_{2} / \mathrm{l}$ ). Des valeurs égalant 4 à 6 fois la limite d'utilisation en agriculture ont été notées avec les eaux du canal aval de la STEP, du bassin de stockage des eaux en aval des parcelles aménagées et du site MACO Tan Aliz. Ces eaux ont aussi eu les valeurs très élevées de la DCO avec plus de 7 fois la limite maximale.

\section{Perception des producteurs sur la qualité des eaux utilisées}

Pour $81 \%$ des horticulteurs interrogés, 
les eaux usées sont utilisées en toute saison. Seulement $6 \%$ de ces acteurs les utilisent uniquement en saison pluvieuse et $13 \%$ en saison sèche. Par ailleurs, il ressort des travaux de terrain que ces eaux sont également utilisées à d'autres fins. En effet, $32 \%$ des horticulteurs ont révélé qu'elles sont aussi utilisées par d'autres usagers notamment pour la confection de briques de construction d'habitations, pour l'abreuvement des animaux, pour l'arrosage des plantations d'alignement et des voies publiques lors de certains travaux de voirie.

La perception des producteurs sur la qualité des eaux utilisées et leurs impacts varie selon leur niveau de vie et d'instruction, le site occupé ainsi que les spéculations produites. Près de $2 / 3$ des horticulteurs $(58 \%)$ ont affirmé que les eaux usées conviennent à toutes les espèces végétales, tandis qu'environ le tiers (27\%) a eu des difficultés de production de certaines espèces notamment les épinards et $15 \%$ ignorent si l'utilisation de ces eaux comporte des dangers pour les plantes.

En ce qui concerne les impacts environnementaux liés à l'utilisation des eaux usées, les manifestations déclarées par les producteurs particulièrement dans la zone aménagée de la STEP sont relatives au changement de la texture du sol, aux mauvaises odeurs du site après l'arrosage, à la mort de certains insectes, aux brûlures constatées sur les racines ou les feuilles des plantes surtout les épinards et l'amarante. Toutefois, $71 \%$ des exploitants pensent que les impacts des eaux usées sur l'environnement sont indirects et $21 \%$ affirment que ces impacts se manisfesteront dans le futur.

Quant aux impacts sanitaires, $87 \%$ des enquêtés ont affirmé être au courant de la toxicité des eaux usées. Alors que 13\% d'entre eux disent n'avoir jamais eu de problème de santé en rapport avec ces eaux ; seulement 7\% de ces enquêtés ont avoué avoir déjà subi des préjudices avec ces eaux usées.

\section{Caractéristiques chimiques des sols}

Les sols concernés par l'étude sont des sols généralement enrichis avec des substrats d'origines diverses. Près de $95 \%$ des substrats utilisés pour la production des plants en pépinière et pour la culture maraîchère sont collectés dans les caniveaux d'écoulement des eaux usées ou les espaces non occupés ou sous les tas d'immondices et/ou sous forme de composts, fumiers, déjections animales, etc. Toutefois, $50 \%$ des producteurs ont utilisé les substrats collectés sur place pour des raisons économiques, $20 \%$ parce qu'ils connaissaient leur valeur fertilisante et $30 \%$ sans raison apparente.

Le Tableau 3 présente les caractéristiques chimiques des sols. A l'exception du site 3, les apports d'eaux usées augmentent la teneur en matière organique des sols sous culture. Dans les sites, la matière organique $\mathrm{du}$ sol a été faible $(<2 \%)$ à l'exception du site du Barrage $\mathrm{n}^{\circ} 3$ Côté Nord, où elle a été normale (2,50 à $3,10 \%)$. Les teneurs en Azote $(\mathrm{N})$ ont été faible $(<1 \mathrm{~g} / \mathrm{kg})$ et ont augmenté de $0,06 \mathrm{~g} / \mathrm{kg}$ à $0,6 \mathrm{~g} / \mathrm{kg}$ par rapport au témoin observé dans les sites de barrage $n^{\circ} 3$ côté Sud et du Canal MACO Tan Aliz respectivement.

Le $\mathrm{pH}$ des sols a varié de presque neutre $(6,6)$ à fortement alcalin $(9,05)$ observés respectivement dans les sites de Ouaga 2000 et de la rive droite de la STEP Kossodo. Dans les sites de la STEP et du Canal MACO et de Tan Aliz, il y a eu une augmentation du $\mathrm{pH}$ par rapport au témoin de 2 unités de $\mathrm{pH}$. Par contre, dans les sites du Barrage $n^{\circ} 3$ et du Canal Ouaga 2000, on a observé une diminution des valeurs du $\mathrm{pH}$ de 0,5 unité.

Comparativement aux parcelles témoins, les teneurs en phosphore et en potassium total ont également augmenté sur l'ensemble des sites à l'exception du site du Barrage nº 3 Côté Nord. 
Perception des producteurs sur la qualité des sols

Les producteurs n'arrivent pas à discerner les effets spécifiques dus aux eaux usées de ceux dus au terreau utilisé. En effet, $42 \%$ des producteurs estiment ne pas savoir s'il y a des sols plus aptes à l'utilisation des eaux usées. Cependant pour pallier à la pauvreté des sols, les cultures sont menées sur des sols enrichis avec notamment les terreaux des caniveaux ou des substrats divers (composts, déjections, etc.). D'une manière générale, il est fait recours à la fumure organique, à des engrais chimiques, de même qu'à des pesticides divers dans $97 \%$ des cas pour améliorer la production chaque fois que de besoin.

Tableau 2: Caractéristiques physiques, chimiques et biologiques des eaux usées utilisées.

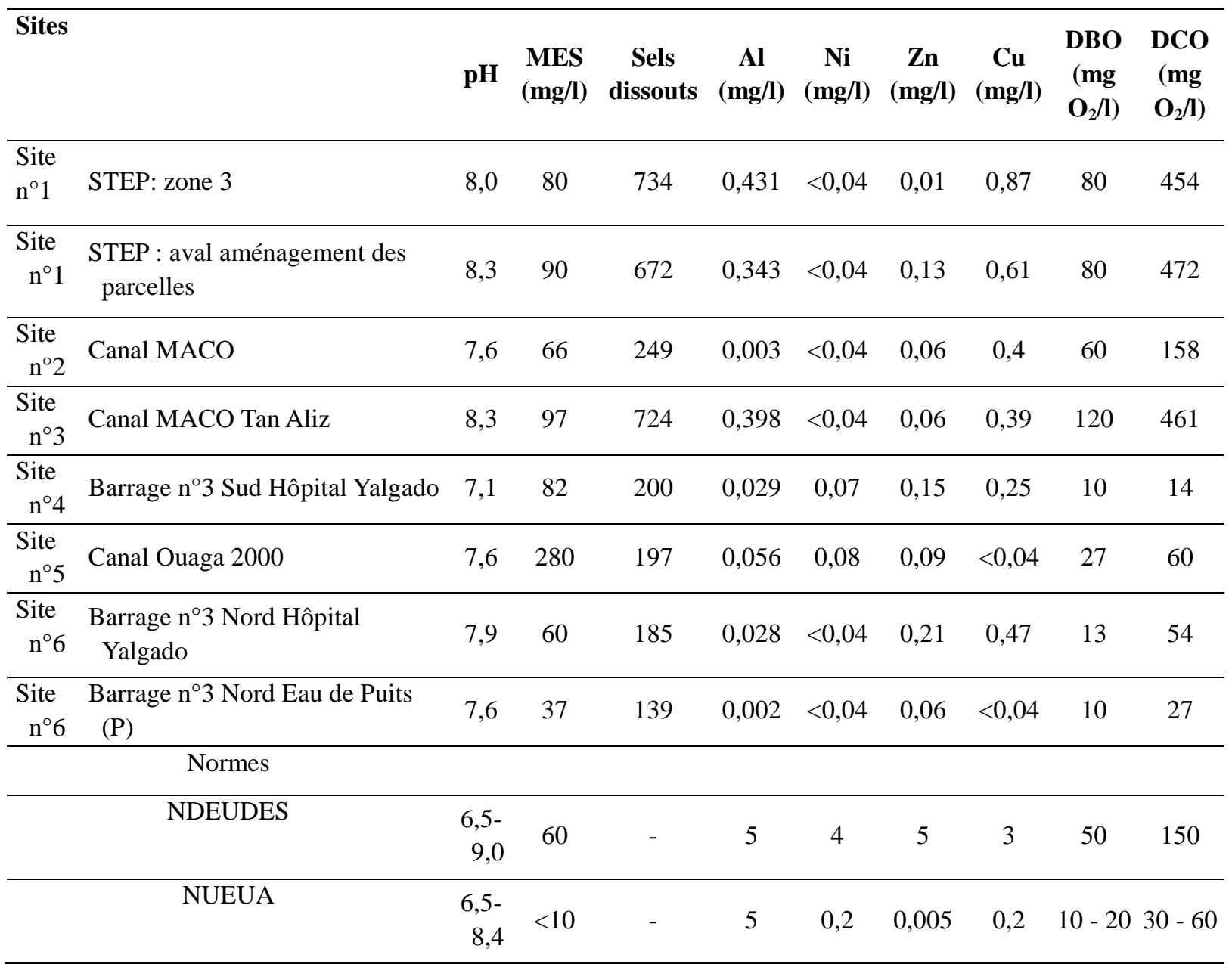

MES: Matières En Suspension.

NDEUDES : Normes de Déversement des Eaux Usées Dans les Eaux de Surface.

NUEUA: Normes OMS d'Utilisation des Eaux Usées en Agriculture. 
Tableau 3: Effets de l'utilisation des eaux usées sur les caractéristiques chimiques des sols.

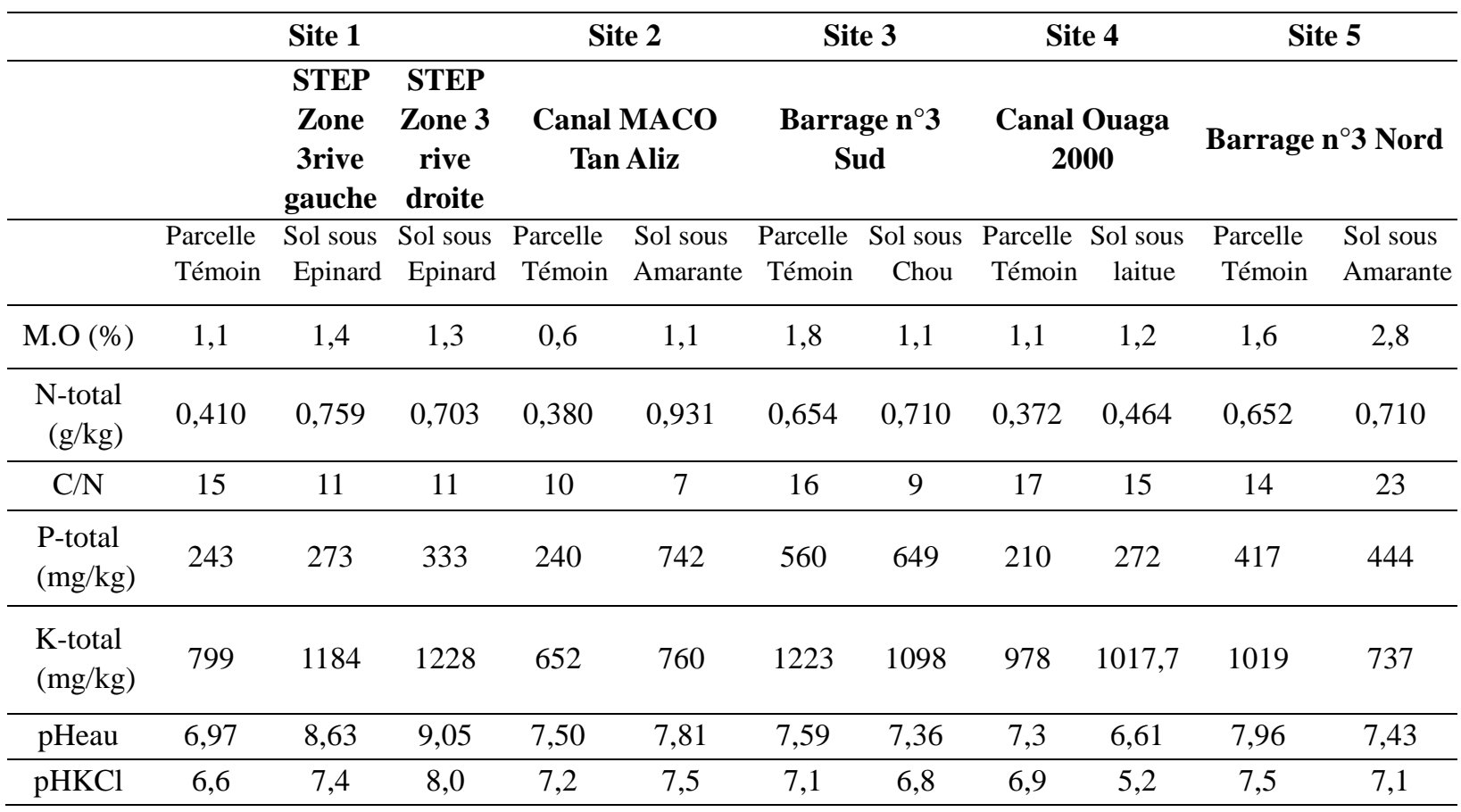

\section{DISCUSSION}

Les systèmes de production recensés autour des sites sont en accord avec les résultats de Boland (2005), qui indique que le contexte urbain offre des opportunités pour la production, l'utilisation de matières premières et la commercialisation dont les populations installées en milieu rural ne disposent pas. En outre, la taille et le lieu de la culture ainsi que la qualité et la quantité de l'eau et du sol sont des facteurs déterminants pour le choix du système de production. Par ailleurs, les horticulteurs perçoivent plus positivement l'utilisation d'eaux usées dans la production de plants que dans la maraîcheculture. Cependant, bien que le risque de contracter des maladies liées à l'usage de ces eaux existent et sont parfois connus, les retombées financières obtenues par les différents acteurs les motivent à persévérer dans ce sens. Ces derniers estiment que les eaux usées ont bien plus de bienfaits que de nuisances. D'où leur insistance dans cette pratique d'utilisation des eaux usées sans aucun traitement ou protection préalable.

Les teneurs élevées en $\mathrm{Cu}$ et $\mathrm{Zn}$ observées sur les sites peuvent s'expliquer par le déversement des eaux usées industrielles. En effet les effluents industriels très chargés en métaux lourds ne sont généralement pas (pré)traités, avant d'être canalisés vers la STEP ou dans les rigoles. Des pollutions similaires ont été rapportées au Cameroun par Kengni et al. (2012). L'utilisation de ces eaux usées chargées en métaux lourds en agriculture, constitue un risque de santé publique et présente en outre des effets néfastes sur les plantes et les animaux. Ces eaux peuvent être à l'origine de la disparition de certaines espèces végétales et/ou animales entraînant ainsi un dysfonctionnement de la chaîne trophique (Gold, 2002).

Quoique le $\mathrm{Zn}$ soit peu toxique, il 
s'accumule très facilement dans les tissus végétaux. Les métaux lourds contenus dans les eaux usées peuvent non seulement polluer la nappe phréatique par infiltration mais aussi nuire à la santé par contact prolongé avec les producteurs. En effet, les maladies les plus fréquemment signalées par les producteurs sont les dermatoses, l'assèchement de la peau, le fendillement des pieds. Des symptômes similaires ont été rapportés par CTA/ETC-RUAF/CREPA (2002) et Christopher et al. (2003). D'autres maladies parasitaires et diarrhéiques ont aussi été rapportées par Kpoda et al. (2015) et ailleurs dans le monde par Dickin (2016).

Au regard de la norme OMS pour la DBO et la DCO, les eaux du barrage $n^{\circ} 3$ et du Canal de Ouaga 2000 ne présentent pas de pollution organique. Cependant, la situation de pollution observée au niveau des eaux de la STEP et du canal de la MACO s'explique par leur non traitement ou traitement insuffisant. Plusieurs études menées ont rapporté des valeurs similaires (Compaoré, 2009; Sawadogo et al., 2012; Compaoré, 2013).

Les $\mathrm{pH}$ des eaux des barrages sont neutres à légèrement alcalins $(7,1-7,9)$ et sont dans la limite tolérable pour la plupart des espèces végétales et animales (5 à 9). Nos résultats corroborent ceux de Lamizana-Diallo (2008) dans le cas du barrage de Massili dont les barrages étudiés sont des affluents et de Ouattara et al. (2012) pour le barrage de Ziga. Les $\mathrm{pH}$ plus basiques $(8-8,3)$ observés au niveau de la STEP et du Canal Tan Aliz peuvent s'expliquer par l'utilisation des produits basiques industriels tels que la soude caustique. Nos résultats sont similaires à ceux rapportés par Sawadogo et al. (2012) et sont par contre plus faibles que ceux rapportés par Zongo et al. (2012).

Les caractéristiques physico-chimiques des sols sont tributaires de celles des eaux utilisées et des pratiques paysannes (Kiba et al., 2012; Mahugua et al., 2017). Les faibles taux de MO sur tous les sites sont à l'instar des sols de la zone. Cette matière organique est rapidement décomposée car le $\mathrm{C} / \mathrm{N}$ des parcelles sous culture est inférieur à 10 .

L'utilisation des eaux usées augmente le $\mathrm{pH}$, les teneurs en carbone, en azote, en phosphore total et en potassium total de ces sols. Cela suggère que les eaux usées peuvent servir de source gratuite de fertilisants, permettant aux producteurs d'économiser sur l'achat des engrais tout en améliorant les productions horticoles.

Cependant, les apports répétés des substrats et des eaux usées peuvent conduire à un excès en azote (Laurent et al., 2011) entraînant des retards de maturation, une altération de la qualité des plantules constatée au niveau de la STEP. L'excès de nitrates peut aussi entrainer une pollution de la nappe phréatique.

L'augmentation excessive des teneurs en $\mathrm{P}$ et $\mathrm{K}$ du sol suite à l'irrigation avec les eaux usées peut non seulement entraver la croissance normale des plantes mais aussi modifier la structure du sol en la rendant plus compacte telle que relevé par les producteurs enquêtés. Cependant, des travaux menés en Algérie par Ababsa et al. (2016) rapportent des effets favorables de l'irrigation avec des eaux usées sur l'amélioration la porosité, la rétention en eau et la conductivité hydraulique mais aussi de l'abondance des communautés lombriciennes.

Egalement, l'excès des phosphates sur l'ensemble des sites entraîne la fixation d'une partie de ces phosphates dans le sol et une autre partie peut être entraînée par l'érosion et participer au phénomène d'eutrophisation.

\section{Conclusion}

Notre étude a montré que le maraîchage et la production de plants sont pratiqués respectivement par $50 \%$ et $35 \%$ des 
horticulteurs. A l'exception des sites de la STEP et de la MACO qui n'ont pratiqué uniquement que le maraîchage et la production des plants en pépinière respectivement, la plupart des sites $(67 \%)$ ont combiné le maraichage et la production des plants en pépinière. Les eaux utilisées sur les sites d'étude sont essentiellement usées dans 90,32\% des cas et ont un $\mathrm{pH}$ compris entre 7,1 et 8,3, des teneurs en $\mathrm{Zn}$ atteignant 40 fois la norme d'utilisation en agriculture, et des DBO et de la DCO atteignant 6 et 7 fois la limite d'utilisation en agriculture.

L'utilisation des eaux usées augmente le $\mathrm{pH}$, les teneurs en carbone, en azote, en phosphore total et en potassium total de ces sols, présageant que les eaux usées peuvent servir de source gratuite de fertilisants. Cependant, ces eaux présentent des risques sanitaires sur les producteurs. Tout comme l'a développé Furedi et al. (2000) in Athséminaire (2002) «la réutilisation doit être acceptable, sûre et économique pour les autorités urbaines, les travailleurs agricoles et les familles». Pour ce faire un dispositif de suivi et d'évaluation périodique de la qualité des eaux et des sols devra être formellement mis en place. Toutefois, la prise en compte des variables telles que le statut social des producteurs, la main d'œuvre, le niveau de connaissances dans les secteurs horticoles, les superficies emblavées, contribuerait d'une part à l'orientation de la vocation des sites de production et d'autre part au choix de systèmes de production des exploitants.

\section{CONFLIT D'INTERETS}

Les auteurs ont affirmé qu'il n'y avait aucun conflit d'intérêt.

\section{CONTRIBUTIONS DES AUTEURS}

DBO est le principal meneur de l'étude. ZG a contribué au suivi régulier du déroulement de l'étude et à la rédaction. HBN et MPS se sont investis dans la lecture et à l'amendement du manuscrit.

\section{REMERCIEMENTS}

Les auteurs remercient Dr Roger KISSOU pour ses observations et critiques du manuscrit.

\section{REFERENCES}

Ababsa N, Kribaa M, Tamrabet L, Addadd D, Zidi A, Mansouri D. 2016. Relation entre l'activité biologique et le comportement hydro physique des sols dans le contexte du système prairial naturel irrigué à l'eau usée. European Scientific Journal, 12(5): 76-89. DOI : http://dx.doi.org/10.19044/esj.2016.v12n $5 \mathrm{p} 76$

ATH Séminaire. 2002. Les avantages de la réutilisation des eaux usées. ATH, (25) : 93-99.

Atidegla SC, Bonou W, Agbossou EK. 2017. Relation entre perceptions des producteurs et surferlisation en maraîchage urbain et périurbain au Bénin. Int. J. Biol. Chem. Sci., 11(5): 2106-2118.

DOI: http://dx.doi.org/10.4314/ijbcs.v11i5.14

Atinkpahoun CNH, Soclo HM, Pons M-N, Leclerc JP. 2018. Physico-chemical characterization of domestic wastewaters in the "Nouvelle" agglomeration, Cotonou, Bénin. Int. J. Biol. Chem. Sci., 12(1): 542-557. DOI : http://dx.doi.org/10.4314/ijbcs.v12i1.42

Bah A. 2014. L'agriculture urbaine en Afrique: un potentiel vivrier sousexploité face à l'insécurité alimentaire. http://www.fondationfarm.org/zoe.php?s=blogfarm\&w=wt\&id $\mathrm{t}=1849$ Consulté le 08/05/16.

Boland J. 2005. Agriculture Urbaine: la Culture des Légumes en Ville. CTA, Série Agrodok (24) : 94. 
BUNASOLS. 1990. Manuel pour l'évaluation des Terres. Documentation Technique $\mathrm{N}^{\circ} 6,181 \mathrm{p}$.

Christine A. 2013. L'agriculture urbaine, contributrice des stratégies alimentaires des mégapoles? Daniel T. 2013. 24èmes Journées Scientifiques de l'Environnement. La transition écologique des mégapoles. Créteil, France. JSE, 9-12.

Christopher SA, Wang J, Ji Q, Tsay SC. 2003. Estimation of diurnal shortwave dust aerosol radioactive forcing during PRIDE. Journal of Geophysical Research, $\quad$ 108(D19): 8596. DOI : 10.1029/2002JD002787

Compaoré M. 2009. Contribution à l'aménagement et la gestion intégrée des services d'eau et d'assainissement de la cité SOCOGIB de Ouaga 2000. Master en ingénierie de l'eau et de l'environnement option : eau et assainissement, Institut International d'Ingénierie de l'Eau et de l'Environnement (2ie), OuagadougouBurkina Faso, 113p.

Compaoré RF. 2013. Gestion des eaux usées et excrétas dans un milieu carcéral en Afrique de l'Ouest : cas de la maison d'arrêt et de correction de Ouagadougou (MACO) au Burkina Faso. Master en ingénierie de l'eau et de l'environnement option : eau et assainissement, Institut International d'Ingénierie de l'Eau et de l'Environnement (2ie), OuagadougouBurkina Faso, 68p.

CTA/ETC-RUAF/CREPA. 2002. Visite d'étude et Atelier International sur la Réutilisation des Eaux Usées en Agriculture Urbaine : un défi pour les municipalités en Afrique de l'Ouest et du Centre, Ouagadougou. Rapport Final CTA, $186 \mathrm{p}$.
Diarra J. 2008. Impact des eaux épurées de la station d'épuration de l'ONEA sur la qualité physico chimique et bactériologique des sols de la zone de maraîchage. Rapport de stage de Licence Professionnelle Sols, Déchets et Aménagement du territoire, IGEDD, UO, 59p.

Dickin SK, Schuster-Wallace CJ, Qadir M, Pizzacalla K. 2016. A review of health risks and pathways for exposure to wastewater use in agriculture. Environ Health Perspect, 124(7): 900-909. DOI: http://dx.doi.org/10.1289/ehp.1509995

Drechsel P, Graffe S, Sonou M, Cofie OO. 2006. Informal Irrigation in Urban West Africa: An Overview. Research Report 102, International Water Management Institute, Colombo-Sri Lanka, 43p.

Fontes J, Guinko S. 1995. Carte de la végétation et de l'occupation du sol du Burkina Faso. Notice explicative, Ministère de la Coopération Française, projet Campus, Toulouse, 68p.

Gold C. 2002. Etude des effets de la pollution métallique $(\mathrm{Cd} / \mathrm{Zn})$ sur la structure des communautés de diatomées périphytiques des cours d'eau. Approches expérimentales in situ et en laboratoire. Thèse de Doctorat, Université Bordeaux I, France, 175p.

INSD (Institut National de la Statistique et de la Démographie). 2015. Annuaire statistique 2014. INSD, Ministère de l'Economie et des Finance, Burkina Faso, 386p.

Jourdan N, Giroux G. 2016. Enquête sur la structure des exploitations agricoles en 2013. Agreste Primeur, 337: 1-6. http://www.epsilon.insee.fr:80/jspui/hand le/1/46634

Kêdowidé-Conchita MG, Sedogo MP, Cissé G. 2010. Dynamique spatio-temporelle de l'agriculture urbaine à Ouagadougou : 
cas du maraîchage comme une activité montante de stratégie de survie. VertigO, 10(2). DOI : 10.4000/vertigo.10312

Kengni L, Tematio P, Filalirharrassi K, Ngueke JT, Tsafack EI, Mboumi TL, Mounier S. 2012. Pollution des eaux superficielles et des nappes en milieu urbain : cas de la zone industrielle de Douala-Bassa (Cameroun). Int. J. Biol. Chem. Sci., 6(4): 1838-1853. DOI : http://dx.doi.org/10.4314/ijbcs.v6i4.39

Kiba DI, Zongo NA, Lompo F, Jansa J, Compaoré E, Sedogo PM, Frossard E. 2012. The diversity of fertilization practices affects soil and crop quality in urban vegetable sites of Burkina Faso. Europ. J. Agronomy, 38: 12- 21. DOI: https://doi.org/10.1016/j.eja.2011.11.012

Kpoda NW, Ouéda A, Somé YSC, Cissé G, Maïga-Amadou H, Kabré GB. 2015. Physicochemical and parasitological quality of vegetables irrigation water in Ouagadougou city, Burkina-Faso. African Journal of Microbiology Research, 9(5): 307-317. DOI: https://doi.org/10.5897/AJMR2014.7295

Laurent F, Dupont N. 2011. L'eau dans le milieu. Université Virtuelle Environnement \& Développement Durable. 64p. http://uved.univlemans.fr/docs/Grain-1.pdf.

Lamizana-Diallo MB, Kenfack, MillogoRasolodimby J. 2008. Evaluation de la qualité physico-chimique de l'eau d'un cours d'eau temporaire du Burkina Faso : le cas du Massili dans le Kadiogo. 2IE, Institut International d'Ingénierie de l'Eau et de l'Environnement. Sud Sciences Technologies, 16 : 23-28. URI : http://hdl.handle.net/2042/30381.

Mahugua JAM, Nambela L, MMochi AJ. 2017. Levels and distribution of pesticide residues in soil and sediments in Easter Lake. Int. J. Biol. Chem. Sci.,
11(5): 2537-2547. DOI: https://dx.doi.org/10.4314/ijbcs.v11i5.46

MINEFID. 2016. Schéma Nationale d'Aménagement et de Développement Durable du Territoire (SNADDT). DGDT, MINEFID, Burkina Faso, 172p.

OMS. 2012. Directives OMS pour l'utilisation sans risque des eaux usées, des excréta et des eaux ménagères : utilisation des eaux usées en agriculture. OMS, 2 : 23-26.

Ouandaogo N, Yameogo S. 2008. Ressources en eau souterraine du centre urbain de Ouagadougou au Burkina Faso. «Qualité et vulnérabilité». Thèse de Doctorat de l'Université d'Avignon, Burkina Faso.

Ouattara Y, Guiguemde Y, Diendéré F, Diarra J, Bary A. 2012. Pollution des eaux dans le bassin du Nakambé : cas du barrage de Ziga. Int. J. Biol. Chem. Sci., 6(6): 80348050 .

DOI: http://dx.doi.org/10.4314/ijbcs.v6i6.47

Ouédraogo DB. 2010. Utilisation des eaux usées dans les systèmes de productions horticoles urbains au Burkina Faso: Analyse de la situation et proposition d'options pour la ville de Ouagadougou. Master en ingénierie de l'eau et de l'environnement option : eau et assainissement, Institut International d'Ingénierie de l'Eau et de l'Environnement (2ie), OuagadougouBurkina Faso, 89p.

Ouédraogo L, Tankoano JP, Traoré I. 2001. Mapping urban and periurban agricultural areas in Ouagadougou, Burkina Faso (practical application of IKONOS-2 "Very High Resolution" satellite imagery). Thesis Report GIRS2001-35, Center for geo-information, Ouagadougou-Burkina Faso, 47p.

Sawadogo R, Guiguemdé I, Diendéré F, Diarra J, Bary A. 2012. Caractérisation physico-chimique des eaux résiduaires de tannerie : cas de l'usine TAN ALIZ à 
Ouagadougou / Burkina Faso. Int. J. Biol. Chem. Sci., 6 (6): 7087-7095. DOI : http://dx.doi.org/10.4314/ijbcs.v6i6.43

Soltner D. 2014. Les Bases de la Production Végétale (Tome 1): Le Sol (26 ${ }^{\text {ème }}$ édition). Sciences et Techniques Agricoles : France, 472 p.

Sou YM. 2009. Recyclage des eaux usées en irrigation: potentiel fertilisant, risques sanitaires et impacts sur la qualité des sols. Thèse, École polytechnique fédérale de Lausanne (EPFL)- Faculté de l'environnement naturel, architectural et construit (ENAC), Suisse, 178p.

SP/CONAGESE (Conseil National pour la Gestion de l'Environnement). 2002. Premier Rapport de l'Etat de l'Environnement au Burkina Faso (REEB). CONAGESE-Ministère de l'Environnement et de l'Eau (MEE), Burkina Faso, 194p.
Tidjani OMM, Issoufou A, Rabani A, Zeinab S. 2018. Contamination potentielle des aliments par des polychlorobiphényles (PCBs) : connaissance du polluant et évaluation de la perception du risque. Int. J. Biol. Chem. Sci., 12(1) : 168-179. DOI:

https://dx.doi.org/10.4314/ijbcs.v12i1.13

Zida Y. 2009. Monographie de la région du Centre. Ministère de l'Economie et des Finances, Burkina Faso, 154p.

Zongo I, Diallo-Koné M, Palm P, Tiemtoré A, Sanogo O, Guiguemdé E, Lapicque F, Leclerc J-P. 2012. Bilan des eaux usées de la ville de Ouagadougou : capacité auto-épuratoire en vue de la production de biogaz. Scientific Study and Research Chemistry and Chemical Engineering, Biotechnology, Food Industry, 13(2): 153-167.

DOI : http://docplayer.fr/40518623 